\title{
An Approach with a Queue System to the Pandemic Period Study ${ }^{*}$
}

\author{
Manuel Alberto M. Ferreira \\ Instituto Universitário de Lisboa (ISCTE-IUL), Lisboa, Portugal
}

\begin{abstract}
Epidemics still happen, being the annual influenza outbreaks examples of such occurrences. To estimate the epidemic period length is imperative because, in this period, it is necessary to strengthen the health care, through extra availability in human and material resources. So, a huge increase of expenses occurs. As a pandemic is an epidemic with a great population and geographical dissemination, more appropriately this happens with the pandemic period. Mainly using results on the $M|G| \infty$ queue busy period, it is presented an application of this queue system to the pandemic period's parameters and distribution function study.
\end{abstract}

Keywords: $M|G| \infty$, busy period, pandemic, cost

\section{The Model}

All through any queue system operation, a busy period is a period that begins when a customer arrives at the system finding it empty, ends when a customer abandons the system letting it empty and there is always at least one customer present.

A $M|G| \infty$ queue system is characterized by the customers arrive according to a Poisson process at rate $\lambda$, receive, upon the arrival, a service which time length is a positive random variable with distribution function $G($. and mean $\alpha$. The traffic intensity is $\rho=\lambda \alpha$.

A pandemic is an epidemic of infectious disease that is spreading through human populations across a large region ${ }^{1}$. Then the $M|G| \infty$ queue can be applied to the pandemic period study, being the parameter $\lambda$, the rate at which people is infected, supposed the infections occur according to a Poisson process. The service time is the time throughout which an infected person stays sick.

For what interests in this work: a busy period is a pandemic period.

Another work on this subject is Ferreira (2014) and can also be seen in Ferreira (2016).

\section{The Pandemic Period}

This paper calls $P P$ the random variable pandemic period length. According to the results known for the $M|G| \infty$ queue busy period length distribution:

$$
E[P P]=\frac{e^{\rho}-1}{\lambda}
$$

\footnotetext{
${ }^{*}$ Acknowledgement: This work is financed by national funds through FCT - Fundação para a Ciência e Tecnologia, I.P., under the project UID/MULTI/4466/2016. Furthermore, the author thanks the Instituto Universitário de Lisboa and ISTAR-IUL for their support.

Manuel Alberto M. Ferreira, Full Professor, ISTAR-IUL, BRU-IUL, Instituto Universitário de Lisboa (ISCTE-IUL). Email: manuel.ferreira@iscte.pt.

${ }^{1}$ For instance, a continent, or even worldwide, see http://en.wikipedia.org/wiki/Pandemi.
} 
whatever is an infected person sickness time length distribution (Takács, 1962). As for $\operatorname{Var}[P P]$, it depends on the whole sickness time length distribution probabilistic structure. Nonetheless, Sathe (1985) established that:

$$
\lambda^{-2} \max \left[e^{2 \rho}+e^{\rho} \rho^{2} \gamma_{s}^{2}-2 \rho e^{\rho}-1 ; 0\right] \leq \operatorname{Var}[P P] \leq \lambda^{-2}\left[2 e^{\rho}\left(\gamma_{s}^{2}+1\right)\left(e^{\rho}-1-\rho\right)-\left(e^{\rho}-1\right)^{2}\right]
$$

where $\gamma_{s}$ is the sickness time length coefficient of variation.

For $\alpha$ and $\rho$ great enough (very intense infectious conditions) since $G($.$) is such that for \alpha$ great enough $G(t) \cong 0, t \geq 0$, the $P P$ distribution function fulfills:

$$
P P(t) \cong 1-e^{-\lambda e^{-\rho_{t}}}, \quad t \geq 0
$$

which can be seen in Ferreira, Filipe, and Coelho's (2014) work.

Calling $N_{P P}$, the mean number of sick people in the pandemic period, if $G($.$) is exponential:$

$$
N_{P P}=e^{\rho}
$$

For any other $G($.$) probability distribution:$

$$
N_{P P} \cong \frac{e^{\rho\left(\gamma_{s}^{2}+1\right)}\left(\rho\left(\gamma_{s}^{2}+1\right)+1\right)+\rho\left(\gamma_{s}^{2}+1\right)-1}{2 \rho\left(\gamma_{s}^{2}+1\right)}
$$

It can be seen in Ferreira and Filipe's (2010) work. Of course, multiplying Equation (4) or (5), as appropriate, by the mean cost of each sick person treatment, it is possible to estimate the mean health care cost owing to the pandemic period.

\section{Mean Number of Pandemic Periods Occurrence in a Time Interval}

After the renewal processes theory (Çinlar, 1975), calling $R(t)$, the mean number of pandemic periods that begin in $[0, t]$, being $t=0$ the beginning instant of a pandemic period, it is possible to obtain (Ferreira, 2005a; 2005b):

$$
R(t)=e^{-\lambda \int_{0}^{t}[1-G(v)] d v}+\lambda \int_{0}^{t} e^{-\lambda \int_{0}^{u}[1-G(v)] d v} d u
$$

and consequently:

$$
e^{-\rho}(1+\lambda t) \leq R(t) \leq 1+\lambda t
$$

which can be seen in Ferreira's (2005) work. If the sickness time length is exponentially distributed:

$$
e^{-\rho\left(1-e^{-\frac{t}{\alpha}}\right)}+\lambda e^{-\rho} t \leq R(t) \leq e^{-\rho\left(1-e^{-\frac{t}{\alpha}}\right)}+\lambda t
$$

\section{Conclusions}

To apply this model, it is necessary the infections occur according to a Poisson process at constant rate. It is a hypothesis perfectly admissible in this kind of phenomena, since they have great geographic spread, and it is considered the mean arrival rate for the pandemic period as the constant rate.

Among the results presented, Equations (1), (2), and (5) in Section 2 and Equation (7) in Section 3 are remarkable for the easiness and also for requiring only the knowledge of the infectious rate $\lambda$, the mean sickness time $\alpha$, and the sickness time variance. The other results are more complex and demand the goodness of fit test for the distributions indicated to the sickness times. 


\section{References}

Çinlar, E. (1975). Introduction to stochastic processes. New Jersey, NJ: Prentice Hall, Inc.

Ferreira, M. A. M. (2005a). M $|\mathrm{G}| \infty$ queue busy cycle renewal function for some particular service time distributions. Proceedings of the International Conference Quantitative Methods in Economics (Multiple Criteria Decision Making XII) (pp. 42-47). Virt, Slovakia.

Ferreira, M. A. M. (2005b). Differential equations important in the $\mathrm{M}|\mathrm{G}| \propto 0$ queue system transient behaviour and busy period. Proceedings of the APLIMAT 2005-4th International Conference (pp. 119-132). Bratislava, Slovakia.

Ferreira, M. A. M. (2014). The pandemic period length modelled through $\mathrm{M}|\mathrm{G}| \infty$ queue systems. Proceedings of the International Conference Quantitative Methods in Economics (Multiple Criteria Decision Making XVII) (pp. 43-47). Virt, Slovakia.

Ferreira, M. A. M. (2016). Some achievements from a statistical queuing theory research. Acta Scientiae et Intellectus, 2(1), 70-83.

Ferreira, M. A. M., \& Filipe, J. A. (2010). Economic crisis: Using $M|\mathrm{G}| \infty$ queue systems busy period to solve logistics problems in an organization. China-USA Business Review, 9(9), 59-63.

Ferreira, M. A. M., Filipe, J. A., \& Coelho, M. (2014). Unemployment modelled through $\mathrm{M}|\mathrm{G}| \infty$ systems. International Journal of Latest Trends in Finance and Economic Sciences, 4(1), 677-679.

Sathe, Y. S. (1985). Improved bounds for the variance of the busy period of an $M|G| \infty$ queue. Advances in Applied Probability, 17(4), 913-914.

Takács, L. (1962). An introduction to queueing theory. New York, NY: Oxford University Press. 